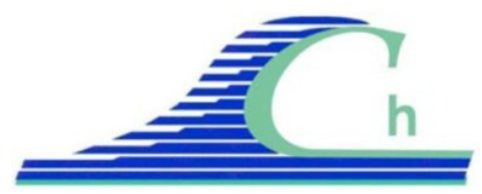

XII ${ }^{\text {èmes }}$ Journées Nationales Génie Côtier - Génie Civil

Cherbourg, 12-14 juin 2012

DOI:10.5150/jngcgc.2012.007-E @ Editions Paralia CFL

disponible en ligne - http://www.paralia.fr - available online

\title{
Laboratory simulation of run-up caused by the resonant waves
}

\author{
Alexander EZERSKY ${ }^{1}$, Nizar ABCHA ${ }^{1}$ \\ 1. Université de Caen, UFR de Sciences, UMR 6143 CNRS - M2C, \\ Esplanade de la Paix, 14032 Caen, France. \\ Alexander.Ezersky@unicaen.fr ; Nizar.Abcha@unicaen.fr
}

\begin{abstract}
:
Run-up caused by harmonic waves excited by wave maker located at some distance from the shore line is studied experimentally. It is revealed that under certain wave excitation frequencies a significant increase in run-up amplification is observed. It is found that this amplification is due to the excitation of resonant mode in the region between the shoreline and wave maker. Frequency and magnitude of the maximum amplification are in good correlation with the numerical calculation results represented in the paper (STEFANAKIS et al., 2011).
\end{abstract}

\section{Keywords:}

Coastal engineering - Run-up - Tsunami - Trapped waves

\section{Introduction}

To characterize the impact of waves on coastal infrastructure, the systematical study of run-up is undertaken. For example, measurements of run-up in natural conditions are carried out after each tsunamis during a long period. As a result, the observations revealed that in some cases, the damage due to a run-up caused by the main wave of the tsunami may be less than that caused by the waves which may exist within a few hours after the arrival of the first wave. Such an increase in the duration of the tsunami can lead to serious consequences for coastal infrastructure. Increasing of tsunami duration is associated with the excitation of trapped (resonant) modes in the coastal zone (NEETU et al., 2011). The existence of such modes is due to the bottom topography. Characteristics of trapped modes have been studied in numerical experiments and in natural conditions (GONZALES et al., 1995), (XANUMA\&TSUJI, 1995). Note that the first theoretical estimates of run-up amplification in the presence of resonance waves were carried out in (KAJIURA, 1978). In a recent paper (STEFANAKIS et al., 2011), a numerical study of the run-up amplification due to trapped waves was performed in very simple model. Run-up was investigated in a one-dimensional model: the excitation of trapped waves in the numerical calculations were modeled by a time-periodic displacement of the free surface of the ocean, given as a boundary condition at a certain distance from the shore. Run-up was studied in a fluid layer of variable depth using well known shallow water approximation. 
The main result of STEFANAKIS et al. (2011) is that at a certain frequency of the waves there exists a significant increase in the amplitude of the run-up. According to calculations, the maximal run-up can be 50 times greater than the free surface oscillation amplitude used in the boundary conditions in the numerical calculations. It should be noted that the estimations presented in different papers provide us an order of maximal run-up much less than in (STEFANAKIS et al., 2011). It was established in (STEFANAKIS et al., 2011) that the wave period for which maximal run-up amplification is observed depends on the slope of the bottom and the depth of water in the place where the waves are excited. This period is much larger than the "natural period" -time needed for perturbations to run from the point of excitation to the shoreline and return back-. Results obtained (STEFANAKIS et al., 2011) pose a lot of questions. That is why in order to investigate the mechanisms of run-up amplification caused by trapped waves, we carried out a physical simulation of this processe in the hydrodynamic channel with a inclined bottom. The simulation is carried out in such a way that its results can be used for comparison with numerical calculations presented in (STEFANAKIS et al., 2011).

\section{Experimental procedure}

Experiments were performed in a long hydrodynamic flume $0.5 \mathrm{~m}$ width. The flume is equipped with a wave-maker controlled by computer. To simulate an inclined bottom a PVC plate with thickness of $1 \mathrm{~cm}$ is used. The plate is placed at different angles relatively to the horizontal bottom of the flume in the vicinity of the wave-maker (see Fig. 1). Two resistive probes are used to measure a displacement of water surface. Probes allow us to investigate the amplitude of free surface oscillations and phase of oscillation (one probe is used as a "clock") along the flume. Run-up is determined by processing a movie which is shot by a high-speed camera mounted as shown in Fig. 1. Wave-maker allows us to excite harmonic wave of a given frequency and it works in two regimes: displacement-control and force-control. It is not possible to control free surface displacement, as it was done in the numerical experiment. That is why to study the run-up amplification, simultaneous measurements of the amplitude of free surface displacement near the wave maker and maximal run-up are carried out for different frequences of exitation. 


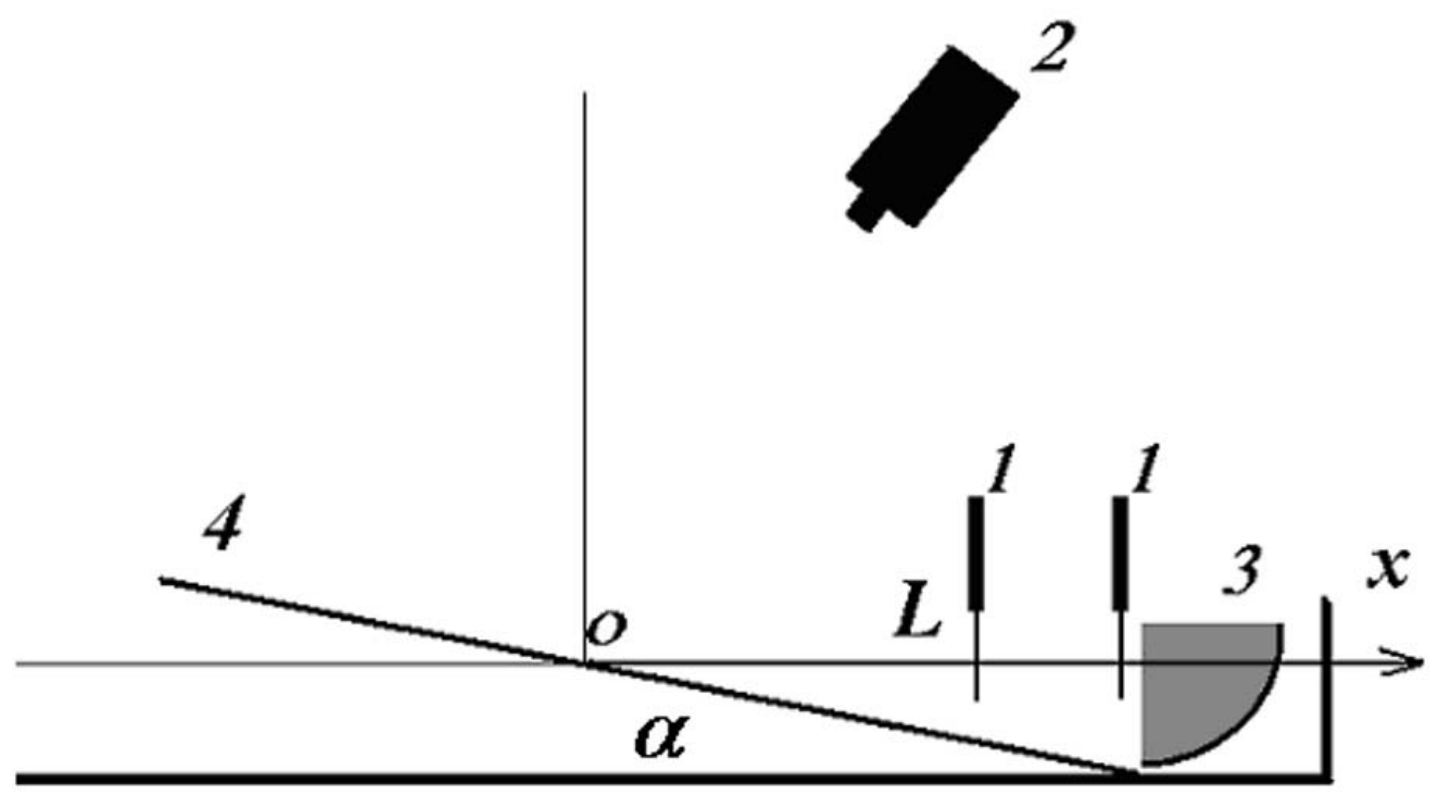

Figure 1. Schema of experiment: resistive probes (1), high-speed video camera (2), wave maker (3), inclined bottom (4).

\section{Experimental results}

Frequency dependence of the amplitude of free surface displacement near the wave maker $(a)$, maximal run-up $(R)$ and coefficient of run-up amplification $(C, C=R / a)$ are shown in Fig. 2 for the slope of the bottom $\tan \alpha=\theta=0,263$. The amplitude of free surface displacement has peaks at frequencies $f_{1}=0.44 \mathrm{~Hz}$ and $f_{2}=0,78 \mathrm{~Hz}$. They are the resonance frequencies of the system. The maximal run-up does have sharp pecks, only small increase in the vicinity of $f_{1}$ and $f_{2}$ is observed (Fig. 2b), but coefficient of run-up amplification (Fig. 2c) increases very sharply in the vicinity of $f_{3}=0.28 \mathrm{~Hz}$ and $f_{4}=0.63 \mathrm{~Hz}$. It is evident that maximal amplification of run-up is observed for frequencies corresponding to the minimal amplitude $a$. In the vicinity of the wavemaker the amplitude is sufficiently small and the signal is very noisy. That is why the coefficient of run-up amplification requires rather delicate measurements of free surface displacement: a band-pass filter was used to measure the amplitude of the harmonic corresponding to wave-maker forcing.

It is important to note that frequency of maximal amplification does depend on the method of wave excitation. Results presented in Fig. 2 were obtained for forcecontrolled regime of wave maker; the same results for coefficient of run-up amplification were obtained for displacement control regime. 


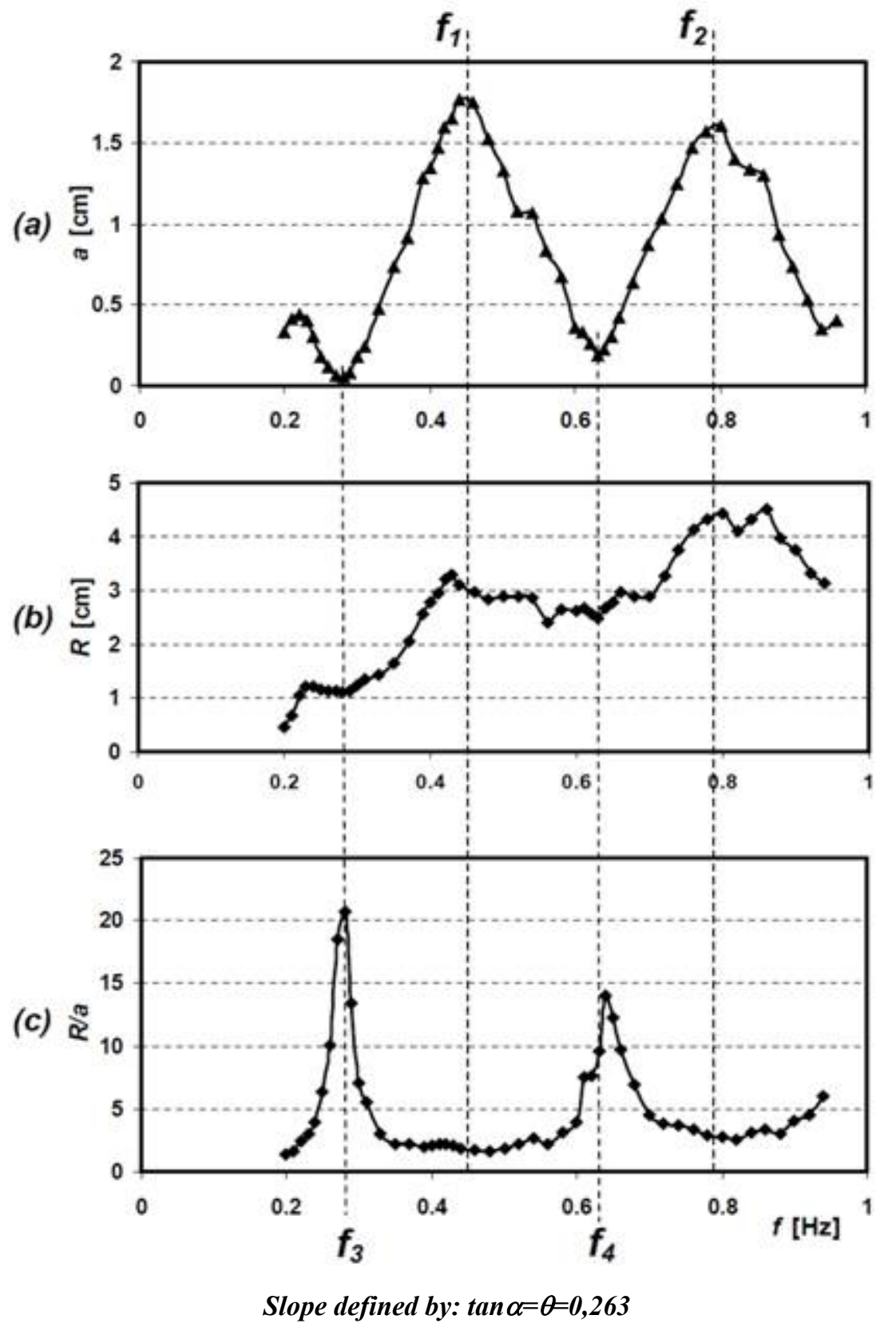

Figure 2. (a) Frequency dependence of amplitude of free surface displacement on frequency (resonance curve). (b) Maximal run-up. (c) Amplification of run-up (ratio of maximal run-up and amplitude of surface wave).

Amplification coefficient $C$ was investigated for three bottom inclinations. Frequencies of maximal amplification depend on angle $\alpha$ and to compare results obtained for different angles $\alpha$, the non-dimensional frequency $F$ was introduced:

$F=\frac{f}{f_{0}}, \quad f_{0}=\frac{1}{K} \sqrt{\frac{g}{H}} \tan \alpha, \quad K=5.23$

where $g$ is for acceleration of gravity, $H$ is for water depth near wave maker 


\section{XII ${ }^{\text {èmes }}$ Journées Nationales Génie Côtier - Génie Civil \\ Cherbourg, 12-14 juin 2012}

Results are presented in Fig. 3. Non-dimensional frequencies of maximal run-up amplification $F=F_{A}=1$ for different angle $\alpha$ coincide very precisely. The coefficient of maximal amplification, corresponding to the frequency $F_{A}=1$ is approximately the same for different inclinations: $C \approx 20 \div 25$. The second peak of run-up amplification coefficient is observed for frequency $F_{B}=(2.2 \div 2.3) F_{A}$. Non-dimensional frequency $F_{B}$ slightly depends on bottom slope; small peak is observed also for frequency $F_{C} \approx 3.5 F_{A}$.

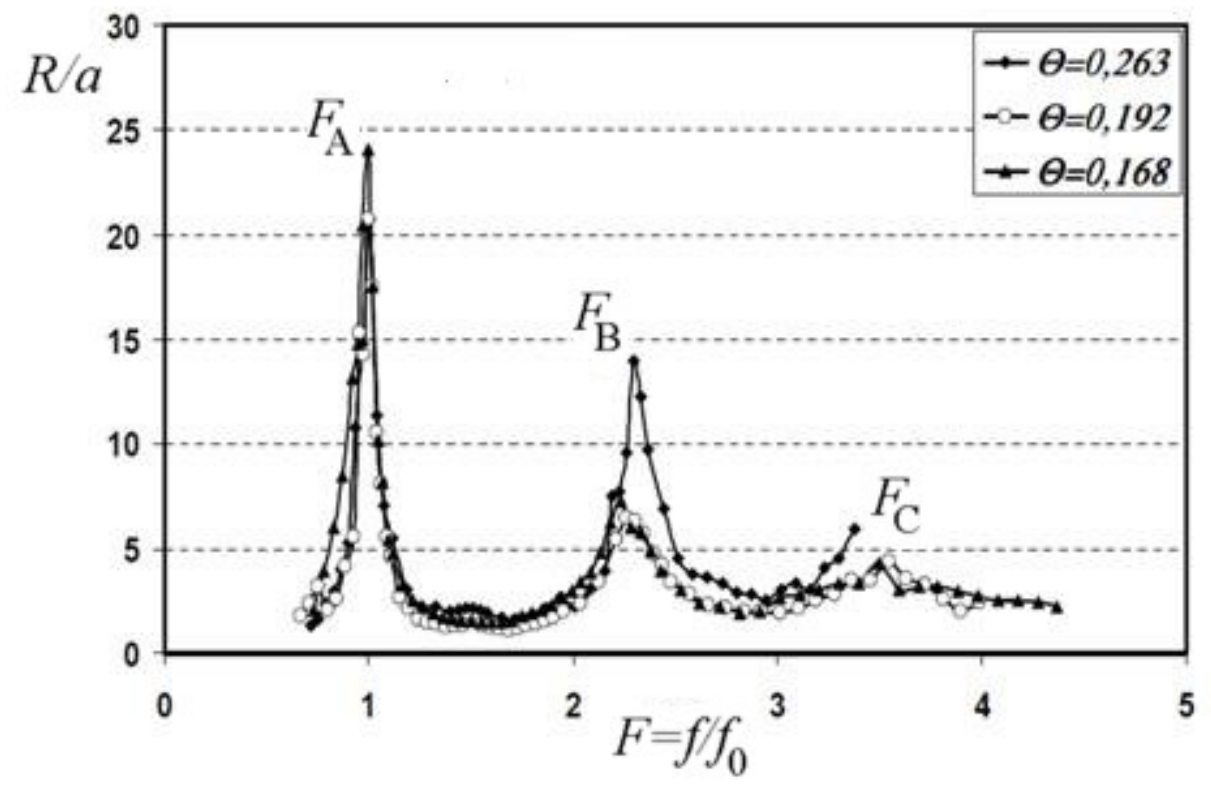

Figure 3. Dependence of run-up amplification on normalized frequency for different bottom slopes.

It should be noted that for our experiemental conditions, linear run-up (run-up without wave bearking) is observed for small frequencies of wave excitation $F<2$, while for higher excitation frequencies $F>2$ near $x=0$ surface wave becomes strongly nonlinear and run-up occurs after the wave breaking. The wavebreaking does not prevent precise determination of maximal run-up position. Excepting high frequencies $F>3$, the border of the water on slope beach was one dimensional and maximal run-up did not depend on coordinate along direction perpendicular to axis $x$.

To study frequency dependence of run-up amplification more precisely, the spatial structures of the free surface oscillations occurring at frequencies corresponding to the resonant frequencies of the system $\left(f_{1}, f_{2}\right)$ and at frequencies of maximum run-up amplification $\left(f_{3}, f_{4}\right)$ have been investigated. The results are shown in Fig. 4 for bottom slop $\tan \alpha=0.168$. Amplitude and phase of free surface displacement are shown by rhombs and circles. Experimental data are compared with well know analytical solution for free surface displacement $\eta$, 
$\eta=J_{0}\left(\sqrt{\frac{4 \omega^{2} x}{g \tan \alpha}}\right) \cos (\omega t)$

This solution was obtained in a shallow water with linear increasing of water depth $H$ : $H=\tan \alpha x$. Theoretical dependences are shown in Fig. 4 by thick lines. The amplitude is chosen as $a=\left|J_{0}\right|$, and $\phi=0$ if $J_{0}>0$ and $\phi=\pi$, if $J_{0}<0$.
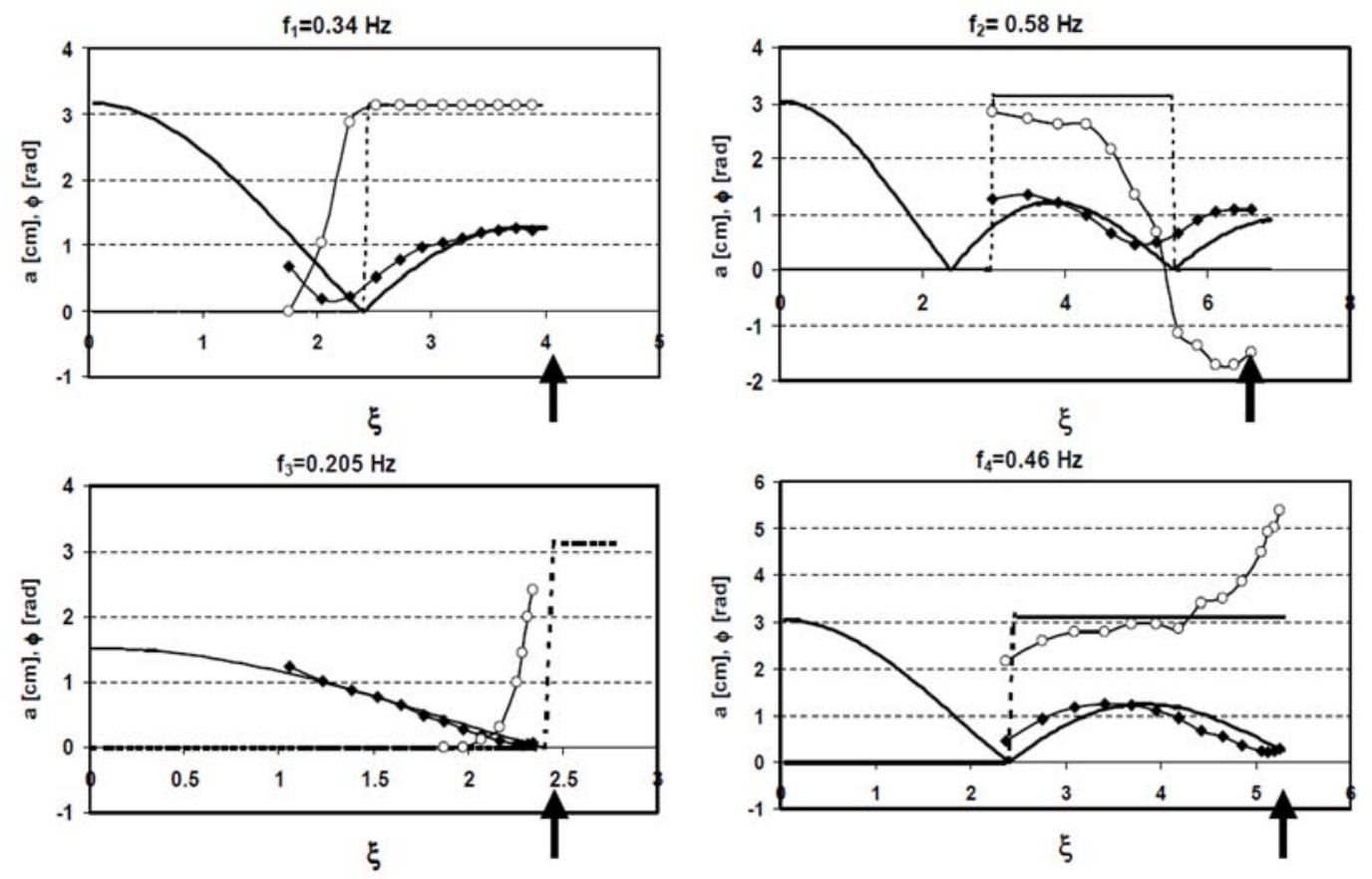

$\xi=\sqrt{\frac{4 \omega^{2} x}{g \tan \alpha}}$. Slope defined by: tan $\alpha=\theta=0,168$. Arrows show positions of the wave-maker edge.

Figure 4. Comparison of experimental values of amplitude (rhombs) and phase (circles) with theoretical values of amplitude (thick solid lines) and phase (thick dash lines) obtained from formula (1).

One can find in Fig. 4 that in the experiment the amplitude does not go to zero and phase changes smoothly for all frequencies. Note that frequencies of maximal run-up amplification ( $f_{3}=0.205 \mathrm{~Hz}, f_{4}=0.46 \mathrm{~Hz}$ ) correspond to spatial modes having minimal amplitude near the wave maker; resonance frequencies $\left(f_{1}, f_{2}\right)$ have maximum amplitude of free surface displacement near the wave maker. It should be noted that according to solution (1), frequencies of maximal run-up amplification correspond to the spatial modes with boundary condition $\eta_{x=L}=0$, and resonant frequencies correspond to mode with boundary conditions:

$\left.\frac{\partial \eta}{\partial x}\right|_{x=L}=0$. 


\section{XII ${ }^{\text {èmes }}$ Journées Nationales Génie Côtier - Génie Civil \\ Cherbourg, 12-14 juin 2012}

In other words, if one uses linear solution (1), the coefficient of run-up amplification in this approximation would be infinite: $a=0$ at $x=L$. In the experiment, the amplitude is small, but finite. Comparison of curves presented in Fig. 4 shows that difference between theoretical solution and experimental data increases with frequency of excitation. For example, these differences are much more significant for $f_{2}$ than for $f_{3}$.

\section{Discussion of results and conclusions}

Let us compare the experimental results with numerical simulations (STEFANAKIS et al., 2011). In the experiment, unlike the numerical calculations, it is not possible to introduce the waves with fixed free surface displacement at definite coordinate. In our opinion this point is not principal. Instead it, the simultaneous measurements of the free surface displacemnt and maximal run-up have bene performed. In our experiment, the frequencies of maximal run-up amplification are very close to those that were obtained in the numerical calculation. We estimated the frequencies of the first peak as:

$$
f_{3}=\frac{1}{K} \sqrt{\frac{g}{H}} \tan \alpha, \quad K \cong 5.23, \quad \text { then: } f_{3}=f_{0}
$$

in (STEFANAKIS et al., 2011) coefficient is estimated as $K \cong 5.1$. Second peak $f_{4}$ in the experimental frequency dependence of run-up is more visible than in numerical simulation (STEFANAKIS et al., 2011). Authors (STEFANAKIS et al., 2011) did not give any estimations of second peak frequency, but if one use their data it is possible to conclude that frequency of the second peak is $2.5 \div 2,7$ times more frequency of the first one. In our experiments the frequency of the second peak exceeds the frequency of first one in $2.2 \div 2,3$ times. Experimental values of frequencies $f_{3-4}$ practically coincide with frequencies of modes having nodes near the wave maker; numerical values exceed this frequency by $2.5 \%$ for all bottom inclinations. The reason of such differences is not clear yet. Non linearity, wave dispersion, viscous dissipation influence the frequency of these peaks, but simple estimations for linear waves in shallow water with zero viscosity provide values which are close to experimental data. Authors (STEFANAKIS et al., 2011) do not mention any dissipation of energy and non -linear parameter, which they use in numerical simulations. As for the coefficient of run-up amplification, the maximal value that was observed in the experiment is $C=20 \div 25$, whereas in (STEFANAKIS et al., 2011) this value reaches $C=50 \div 60$. The difference is apparently due to viscous dissipation, which is essential in our experiments.

Based on the experiments we can conclude that the value of amplification coefficient and frequencies, at which run-up amplification maxima are observed, correlate with results of numerical simulations. The most important conclusion is that the existence of an abnormally large increase of the coefficient $C$ is due to resonator modes: this coefficient becames very large because for its determination the amplitude at the mode node is taken as the amplitude of free surface displacement. It means that numerical 
results of (STEFANAKIS et al., 2011) cannot be applied directly for explanation of runup amplification due to tsunamis: in the natural condition the amplitude of wave packet propagating toward the shore (not amplitude at node) is used to find the coefficient $C$.

\section{References}

GONZALES F.I., SATAKE K., BOSS E.K., MOJELD H.O. (1995). Edge waves and non-trapped modes of 25 April 1992 Cape Mendocino tsunami. PAGEOPH 144, pp 409-426. doi:10.1007/BF00874375

KAJIURA K. (1977) Local behavior of tsunamis. In D. Provis and R. Radok, editors, Waves on Water of Variable Depth, v. 64 of Lecture Notes in Physics, pp 72-79. Springer Berlin/ Heidelberg.

NEETU S., SURESH I., SHANKAR R., NAGARAJAN B., SHARMA R., SHENOI S.S.C., UNNIKRISHNAN A.S., SUNDAR D. (2011) Trapped waves of the 27 November 1945 Makran tsunami: observation and numerical modeling. Nat. Hazards 1, Vol. 59, pp 1609-1618. doi:10.1007/s11069-011-9854-0

STEFANAKIS T.S., DIAS F., DUTYKH D. (2011) Local run-up amplification by resonant wave interaction. Phys.Rev. Lett. 107, 124502. doi:10.1103/PhysRevLett.107.124502

YANUMA T., TSUJI Y. (1998). Observation of Edge Waves Trapped on the Continental Shelf in the Vicinity of Makurazaki Harbor, Kyushu, Japan. Journal of Oceanography, Vol. 54, pp 9-18. doi:10.1007/BF02744377 